\title{
CONSTRUCTION AND ARRANGEMENT OF BRIDGE DEFORMATION JOINTS WITH ELASTIC COMPENSATOR
}

ELENA OVCEARENCO

ROAD DESIGN ENGINEER

\section{TECHNICAL UNIVERSITY OF MOLDOVA}

Abstract. A deformation joint cover device is an assembly designed to safely absorb the temperature-induced expansion and contraction of construction materials, to absorb vibration. It is one of the most important part of a bridge construction, and unfortunately it is not given enough attention to them, what leads to higher repair cost of other parts of the "Art of Work".

Keywords: Deformation joint, joint cover devices, elastic compensator, displacement.

\section{Construction and arrangement of deformation joints with elastic compensator}

Joint cover devices with elastic compensator - is the largest and most diverse range of devices currently manufactured. This type of device, they were originally produced and spread rapidly internationally, and in the early 1960s Europe, began manufacturing modular devices that were absorbing large displacements of superstructures [1].

At present, elastic compensating devices are more advantageous for absorbing medium and large displacements that take place in all directions.

Based on Mr. Mihai Netid researches "Expansion-deformation joints calculation system for bridges with simply supported superstructures, admissible tolerances" and "General trends in the execution of deformation joints at the road bridges with the simple supporting elements superstructure" published in "Problems and innovations in road infrastructure",[2], I've been able to better analyze this type of device with elastic compensator and to write an article about, because the factors which affect the road bridge superstructure are well explained in the book. It is very 
responsible adopting the right choice of joint cover device, that is why are needed more studies on them, because the bridge strength depends if was chosen the best device. Mr. Mihai Netid also describes other types of devices which can be used at building new bridges or rehabilitating the existing ones with concrete superstructure and infrastructure, which in my opinion is helping other scientists to discover and implement new technology and materials for a longer exploitation of them.

\section{Supported displacements.}

Displacements, supported by elastic compensator joint devices, depend on each type. In practice, it is rarely used devices with horizontal-longitudinal displacements less than $25 \mathrm{~mm}$. Typically, the simplest constructions provide displacements ranging from 5 to $40 \ldots 80 \mathrm{~mm}$. The limit displacements of the elastic compensator joint deformations can be made in the longitudinal direction up to $250 \mathrm{~mm}$ and more. The upper limit for such constructions has not yet been established. The elastic deformation device with compensator, provides displacements in the horizontallongitudinal direction up to $600 \ldots 800 \mathrm{~mm}$ and more, and the vertical ones can exceed $50 \mathrm{~mm}$. At the same time, the bridge construction angle is usually assumed to be $45^{\circ}$.

\section{Analysis of joint cover devices with elastic compensator}

Specification of the common construction details of the analyzed devices consists in the absorption of superstructure displacements due to elastic deformations specifically designed for this element - compensator [1]. Compensators can be loadbearing, in this case, apart from the role of absorbing the movements, it carries loads from the vehicle. Non-loader compensators, unlike carrier ones, are used only with the load-bearing elements separated by them, intended for the absorption and transmission of the loads.

Besides this, deformation joints can be constituted with a compensator, constructively executed as flat and bulky profiles (for which there are quite enough thin walls and high elasticity; the profiles may be load bearing and non-load bearing, 
preventative compressed at mounting and uncompressed), or built in the form of monolithic blocks (usually, they are load-carrying compensators, which can be armored for better absorption of loads).

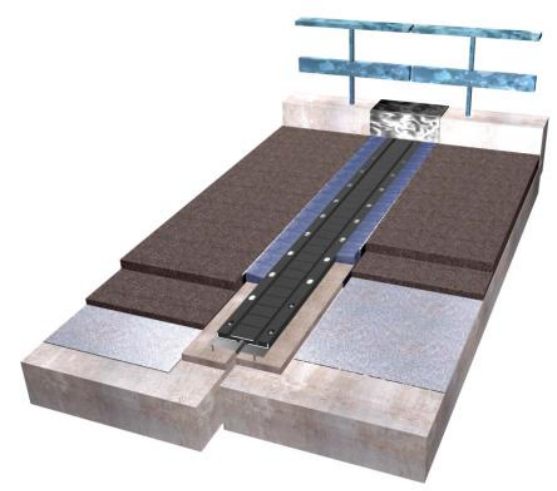

Figure 1. Joint cover device with elastic compensator
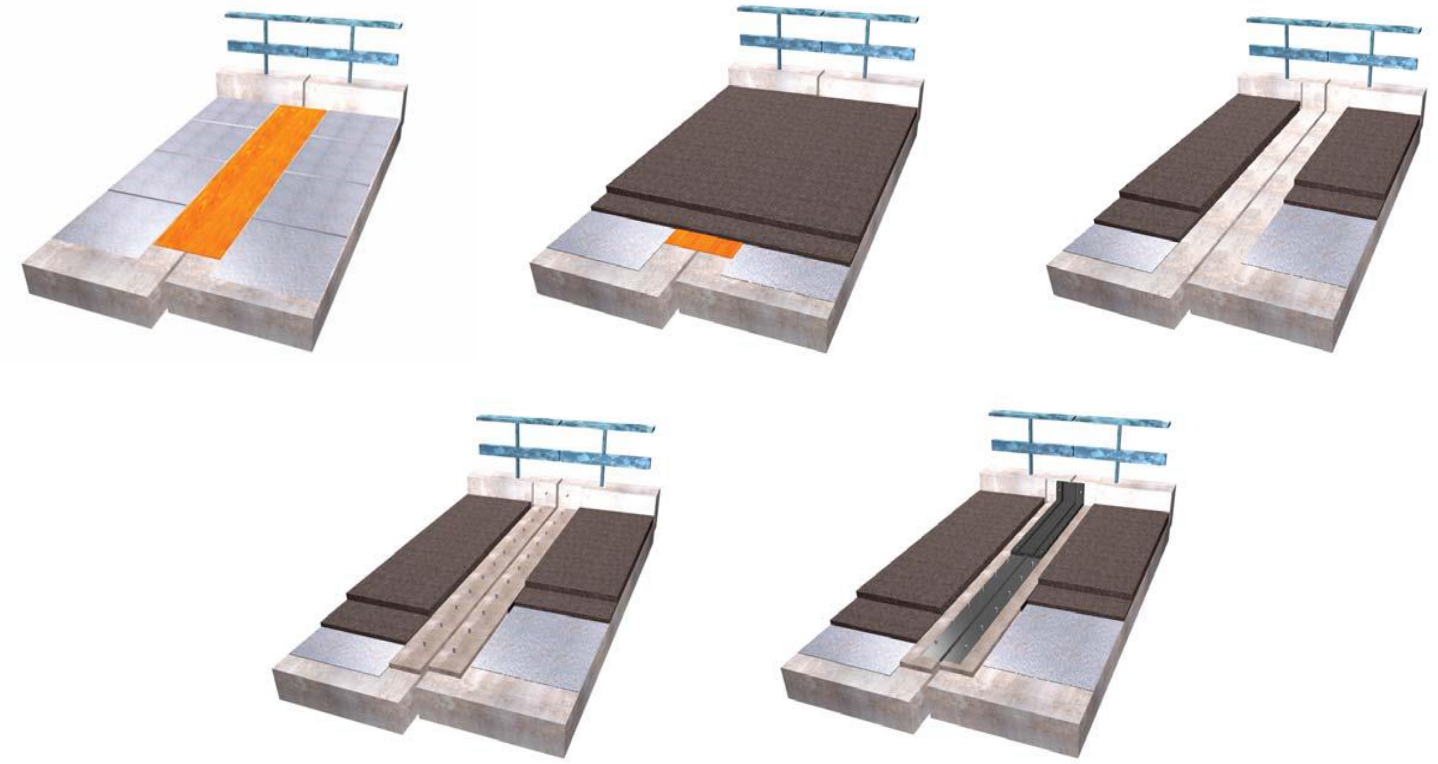

Figure 2. Stages of construction of the joint cover device with elastic compensator

For each type of device with elastic compensator the specificity of the work of the elastic material of the compensator differs. In this way, the compensating material can work at bending, compression, expansion, shearing, even at twisting or at a certain set of deformations listed

Classification 
The elastic compensator deformation joint is classified into simple and multiprofile profiled devices [3].

Simple profiled devices can be of the following types:

- Empty profile and non-bearing;

- With monolithic and casted carrier compensation;

- With monolithic and reinforced carrier compensation;

- With non-bearing tapered profile.

In addition, the devices may be elastic, fixed:

- With adhesive;

- Vulcanized;

- From preventive press (bare profile only);

- By mechanical methods.

By the type of association of superstructure devices, their elastic profile construction can be:

- With metal anchoring elements, reinforced by reinforcement of reinforced concrete superstructures;

- Anchored in concrete reinforced concrete superstructures (with pins or screws);

- Anchored in concrete form with polymers, welded to metallic superstructures;

- United by superstructures with screws.

Joint cover devices may have two types of anchoring from the start or combined.

According to the type of the cross section of the elastic profile, the empty profile devices can be delimited in the constructions of the type:

- Cassette profile; with sectioned profile;

- Profile with cellular structure (mesh). 
Elastically casted devices may have different load carrying offsets. According to the type of upper surfaces of these compensators, the casted ones can be:

- Profile with concave curved surface;

- Flat surface profile.

A jointing device with reinforced and load-bearing monolithic compensators can be divided into the following types (by type of reinforcement):

- With simple profile (with reinforcement of the compensator's support at the bottom);

- With a complex profile (with composite reinforcement of the profile at the top and bottom);

- With external reinforcement of the outer area of the complex profile (with metal surface).

The tapered joint jointing devices are divided by the type of compensator into:

- Cuneiform (wedge-shaped);

- With concave semicircular shape; with convex semicircle shape;

- With the complex section of the profile.

Multiple profile devices are, in fact, multi-profile or modular. Modular devices can and are formed from deformable modules based on any type of single-profile device described previous.

After the location of the deformation modules of the device construction it is divided into:

- Device with module location above the joint;

- Device with out-of-board modules on the superstructure plate.

Depending on the type of work, the bearing elements can be divided:

- Multi-arm construction;

- Single-arm construction; 
- By using as a support for the deformation modules of superstructures (surface mounted module).

According to the principle of organizing the regularization of the uniform distribution of the movements between the deformation modules of the jointing devices, could be:

- Regulating system with elastic connections in case of construction with single support (between bearing beams);

- Regulating system with elastic connections between the adjacent beams in the case of multiple supports;

- Regulatory system with elastic connections between the infrastructure ruler and the superstructure beams in the case of multiple supports;

- Kinematic regulation system.

\section{Analysis of constructive details and experience of use}

One of the criteria that determines the safety of the elastic compensating joints is the way of fixing the compensator used in their construction by the load-bearing elements of the device (or the superstructures of the bridge construction), but also the specificity of the work of the compensator (on which in some measure affects fixation) [3].

At the present stage for most elastic compensator joints, the mechanical method of profile fastening is used. This is due to some disadvantages of other known methods.

Disadvantage of the preventive compressed compensators - need for strong tightening of the compensator to device elements or superstructures. Normal compressional tensions should not be less than $10 \mathrm{~kg} * \mathrm{f} / \mathrm{cm}^{2}$, that is, the sizes, which ensure compact compression of the profile and the tightness of the devices during the operation of the compensators (considering the relaxation of the compressive stresses in the compensator material) [4]. Also, in this case it is difficult to ensure the 
tightness of the devices and the immobility of the compensator according to the project data, in particular the action of waste and mud on it, compacted by vehicle tires.

Bonding of the metal rubber excludes the need for preventive compression of the compensator. That is why the adoption of the dimensions and forms of the compensator will be pursued, which will ensure the transmission of the tasks on the heads of the minimal reactive forces, for example, to the rubber bending work. In the case of cold welding, the preventive compression of the compensators is intended to determine in such a way that glue does not transmit the expansion forces. In the case of "hot" bonding and vulcanization variable voltages are allowed, which will allow the use of large displacement compensators.

Based on international experience, the most favorable working regime of the rubber compensator is to work on bending or bending with expansion [4], although this is not characteristic for all types of elastic compensating joint devices.

As mentioned, the specificity of the compensator work is closely related to the way of fixing them. The characteristic data of this link are shown in Table 20 [4]. Here, the forces are transmitted, which are transmitted on the construction of the joint cover devices for each case.

\section{Conclusions and requirements of constructions.}

To summarize the above, one can draw a series of conclusions regarding the construction of the analyzed devices with elastic compensators:

- It is necessary to perform a clear division and limitation of the ranges of use of the devices with elastic compensator depending on the type of the deformation joint;

- The bare cover jointing device and the concrete ribbed ends (polymer concrete) must be used at the same intervals of displacement and conditions, as in the case of spliced joint cover devices, as a variant alternative of the latter; 
- The elastic and hollow joint jointing device, and mechanical profile fastening, is intended to achieve polymer-backed fins with ribbed properties up to the concept of the device. However, it is necessary to review the method of fixing the compensator in order to prevent it from falling into the channel and increasing the tightness of the device. In addition, it is necessary to reduce the range of use of these types of construction with maximum possible values of space between the limits of the $65 \mathrm{~mm}$ load-bearing elements and to exclude the direct contact of the transport tires with the elastic profiles;

- The cellular or mesh jointing device (including the cassette type) will need to be similar to elastic and hollow profile and mechanically fixed to the profile, and consequently to similar structures;

- The monolithic and cassette-type joint cover device will be used in bridges with complex complexity of superstructure work, in transition areas with surfacing with different surfaces and profile breaks, as well as in sidewalk areas, where such construction works well. It is not advisable to use monolithic and cassette-type devices for increased road traffic (in the roadway area);

- It is necessary to refuse entirely the use of monolithic reinforced loadbearing devices in bridge constructions on account of their low operating properties in favor of single and modular profiles (with 2-3 deformational modules) devices with non-loader elastic compensators;

- Large-scale devices with self-ripped cuneiform tape profiles can be widely recommended, arranged according to the device construction concept, preferably to limit the maximum width of the open joint to 65 $\mathrm{mm}$. In the sidewalk areas it is necessary to use the joint cover, or to use a flat surface device system, for example, a cassette type;

- Tape-shaped modular joint cover devices require measures to ensure flatness for traffic and noise reduction. In the construction of urban 
bridges, the use of measures to reduce noisy emissions is mandatory. Within the urban locality, wherever possible, it is recommended to use "tooth" devices as an alternative. However, it is necessary to mention the limitation to which the use of the toothed structure is related.

One of the best solution of joint cover devices for medium length concrete slabs is described in the book published by Mr. Mihai Netid as "Buried" deformation joint cover devices. These types of devices produce no shock at joints ensuring flatness, which is very good because the dynamic actions from wheels that act on the infrastructure are disregarded and the life expectance of the bridge is automatically increased. Mr. Mihai Netid wrote about many devices which are "Buried", but in my opinion, deformation joint cover device with rubber compensator type "RDC-1-10" (Figure 1.3) [2] because it includes also a drainage system located under pavement and the roadway has a plane surface which does not affect the infrastructure. This type of technology is very useful, but it must be mounted very carefully by the workers and should be monitored by a supervisor during the process because once is "buried", it cannot be checked after if it was right installed. Not least, must be taken into consideration that drivers will also enjoy non-visible joint cover devices during their trips.

\section{References:}

1. Road Bridge deformation joints. Properties of construction and exploitation. A.Efanov, I.Ovchinikov, V.Shesternikov, V.Makarov. Saratov, Russian Federation. 2005 - 94 pages.

2. M. Netid. Problems and Innovations in Road Infrastructure. 2019.

3. Road bridge joints: Design features and work. A. V. Efanov; I. G. Ovchinikov; V. I. Shesternikov; V. N. Makarov. Saratov. Russian Federation 2005 - 94 pages.

4. Expansion joints in road bridges. V. I. Shesterikov. USSR 1978. 151 pages. 